\title{
An isotropic earth field scalar magnetometer using optically pumped helium 4
}

\author{
C. GUTTIN, J.M. LEGER and F. STOECKEL*
}

\author{
LETI, CEA-Technologies Avancées, DSYS-CEN/G, 85X, 38041 Grenoble, France \\ * Laboratoire de Spectrométrie Physique, U.J.F, BP. 87, 38042 St Martin d'Hères, France
}

\begin{abstract}
Optical pumping was originally suggested by Kastler [1] in 1950 and since the early 1960's numerous helium magnetometers based upon that technique have been developed. Since then the main evolution has consisted in the advent in 1986 of tunable lasers suitable for helium optical pumping. They led to a renewed interest in helium magnetometry essentially because the magnetometer resolution is expected to significantly increase with the optical power.

For airborne applications however, these improvements must be associated with a reduction of the anisotropy inherent to optically pumped helium sensors in order to be useful. Our group has therefore recently designed a new helium magnetometer structure which suppresses the sensor orientation effects. To achieve this we perform both the laser optical pumping and the RF resonance excitation in a fixed configuration whatever the sensor orientation with respect to the magnetic field to be measured is.This is performed by rotating simultaneously the laser linear polarization and the RF field direction to maintain them perpendicular to the static field.

In this paper, we explain the principles of operation of such a magnetometer and describe this new sensor which drastically reduces the amplitude and frequency anisotropies of standard helium pumped resonance magnetometers. Finally, we present the preliminary sensor isotropy test results which show a perfect amplitude isotropy and frequency effects smaller than $150 \mathrm{pT}$.
\end{abstract}

\section{Introduction}

Since the construction in the 1960's of the first helium magnetometers based on the optical pumping technique suggested by Kastler [1] in 1950, the main evolution has consisted in the advent of tunable lasers suitable for helium optical pumping. They led to a renewed interest in helium magnetometry mainly because the magnetometer sensitivity is expected to increase with the light source optical power. Moreover, the possibility to tune the laser to the $2^{3} \mathrm{~S}_{1}-2^{3} \mathrm{P}_{0}$ transition of helium 4 allows to build up a higher metastable state polarization as compared with lamp pumping: it has indeed been shown that the relative contributions to the metastable polarization of two out of the three spectral lines emitted by helium discharge lamps around $1083 \mathrm{~nm}$ tend to cancel each other, thus greatly degrading the optical pumping efficiency [2]. The last advantage consists in the possibility to inject the laser beam into fibers, allowing to remove the sensor head further from magnetic perturbations (electronics, light source).

For airborne applications, it is however necessary to reduce the heading errors inherent to helium sensors in order to be able to take full advantage of these improvements. As a matter of fact, if these sensors are not properly oriented in the magnetic field, the resonance signal usually fades away so that no field measurement can be carried out. It consequently represents a serious limitation to the use of such magnetometers for mobile applications. In this paper, we describe firstly the general structure of helium magnetometers and put emphasis on their drawbacks. Then we explain why the new structure that we propose highly reduces the amplitude and frequency anisotropies of standard helium pumped resonance magnetometers. Finally, we present the isotropy test results of our sensor. 


\section{Principles of operation and description}

Helium magnetometers are based on an electronic magnetic resonance whose effects are amplified by a laser pumping process.

The first step is to excite a fraction of the helium atoms (1) to the $2^{3} \mathrm{~S}_{1}$ metastable state by means of a high frequency discharge (2). This energy level is split by the static magnetic field $\mathbf{H}_{\mathbf{0}}$ into three Zeeman sublevels (figure 1). The measurement of their energy separation provides then a very convenient means to determine the earth field.

This is performed thanks to a magnetic resonance experiment. Hence, the second step is to induce transitions between these sublevels in order to detect the resonance. If the applied radiofrequency field (3) matches the Larmor frequency of the Zeeman sublevels, transitions between these sublevels occur and tend to equalize their populations. However, the resonance signal amplitude is very low since at thermal equilibrium the sublevels are almost equally populated and no significant change subsequently results from the resonance.

So the third step is to modify the repartition of the atoms within the three sublevels (alignment or polarization of the metastable state).This is accomplished by optically pumping helium atoms with a tuned laser (4): atoms in the $2^{3} \mathrm{~S}_{1}$ metastable state absorb the laser light with different probabilities for each sublevel and are thus selectively excited to the ${ }^{3} \mathrm{P}_{0}$ state. From there, they undergo a spontaneous emission back to the metastable state. Thanks to this process, the resonance signal amplitude is therefore enhanced by several orders of magnitude.

The resonance can be detected either by optogalvanic techniques or by monitoring the transmitted laser intensity (5) : when the resonance condition is met, the polarization of the metastable state created as a result of the optical pumping process is reduced by the RF field so that the helium cell transparency accordingly decreases. The RF field frequency is then phase locked by an electronic loop to the Larmor frequency, resulting in a field/frequency transducer which can be used as a high sensitivity magnetometer.

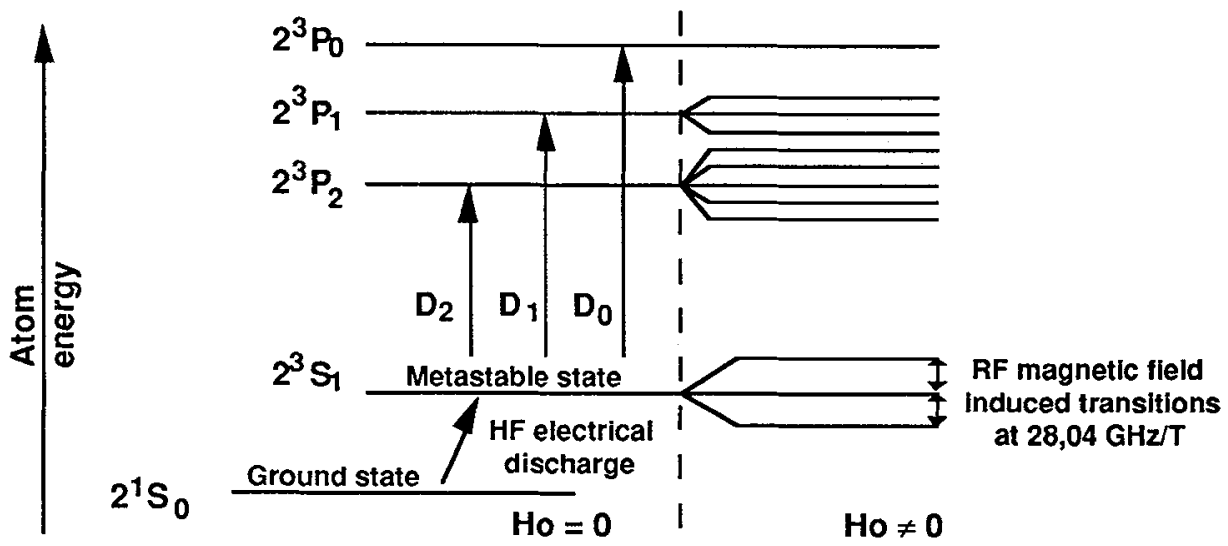

Figure 1: Helium 4 energy levels 
However, the non Boltzman distribution created by optical pumping depends drastically on the relative orientation $\theta_{\mathbf{f}}$ between the laser polarization $\mathbf{E}_{\mathbf{O}}$ and the static magnetic field $\mathbf{H}_{\mathbf{0}}$. In particular for specific directions the optical pumping cycle does not lead to any kind of redistribution of the atoms among the three sublevels, so that the resonance is virtually undetectable. This is the main drawback common to all optically pumped magnetometers.

In addition, only the component of the radiofrequency field $\mathrm{H}_{1}$ perpendicular to the static magnetic field $\mathbf{H}_{\mathbf{O}}$ induces radiofrequency resonant transitions between the $2^{3} \mathrm{~S}_{1}$ Zeeman sublevels. It therefore results in another signal amplitude anisotropy depending on the angle $\theta_{\mathbf{b}}=\left(\mathbf{H}_{\mathbf{1}}, \mathbf{H}_{\mathbf{o}}\right)$.

These phenomena both affect the resonance signal amplitude and consequently the magnetometer resolution.

Besides, the resonance frequency can also be modified due to the interaction of the metastable atoms with either the laser light or the radiofrequency field. Optical pumping for instance generates energy shifts of the Zeeman sublevels whenever the laser wavelength is not exactly equal to the pumping transition center wavelength. As for the RF excitation, the Bloch-Siegert effect [4] results similarly in resonance frequency displacements. Since these two effects depend on the sensor orientation, they give rise to frequency anisotropies of the magnetometer when it is displaced .

\section{Description of the isotropic sensor}

Many methods have already been tested to avoid the resonance signal extinctions. For example, Texas Instruments [5] developed a sensor comprising three cells disposed orthogonally, so that always at least one cell delivers a resonant signal of sufficient amplitude. Our group has recently designed a new helium magnetometer structure presenting no sensor orientation effects made of a single helium cell, thus reducing the sensor head size and simplifying the required electronics.

This method takes advantage of the fact that the resonance signal amplitude is maximum if the laser beam linear polarization $\mathbf{E}_{\mathbf{O}}$ is perpendicular to the static magnetic field $\mathbf{H}_{\mathbf{0}}$ to be measured: it can easily be shown that whatever the direction of the field $\mathbf{H}_{\mathbf{0}}$ is, there always exists a polarization $\mathbf{E}_{\mathbf{0}}$ fulfilling the aforementioned condition for any given light propagation direction $\mathbf{K}_{\mathbf{0}}$. So, in order to create a distribution within the three metastable state sublevels which does not depend on the static field direction, we just have to maintain $\theta f=90^{\circ}$ by adjusting the laser polarization.

This is performed by an amagnetic piezoelectric motor [6] rotating the linear polarizer until it locks perpendicular to the static magnetic field $\mathbf{H}_{\mathbf{0}}$. Moreover, the use of a linear polarized laser beam suppresses the frequency shifts caused by laser detuning, thus slightly reducing the required performances of the laser wavelength control.

As for the radiofrequency field $\mathbf{H}_{1}$, only its component perpendicular to the static field effectively induces resonant transitions between the Zeeman sublevels. Therefore the resonance is most efficiently excited when the condition $\theta \mathrm{b}=90^{\circ}$ is satisfied. As a result, the resonance frequency shift due to the Bloch-Siegert effect is maximum but constant. A potentiometric sensor measuring the angular position of the polarization vector $\mathbf{E}_{\mathbf{o}}$ is used to drive the radiofrequency field direction by means of two orthogonal sets of Helmholtz coils.

By combining these two conditions, we obtain an isotropic helium magnetometer whose structure is presented on figure 2. 


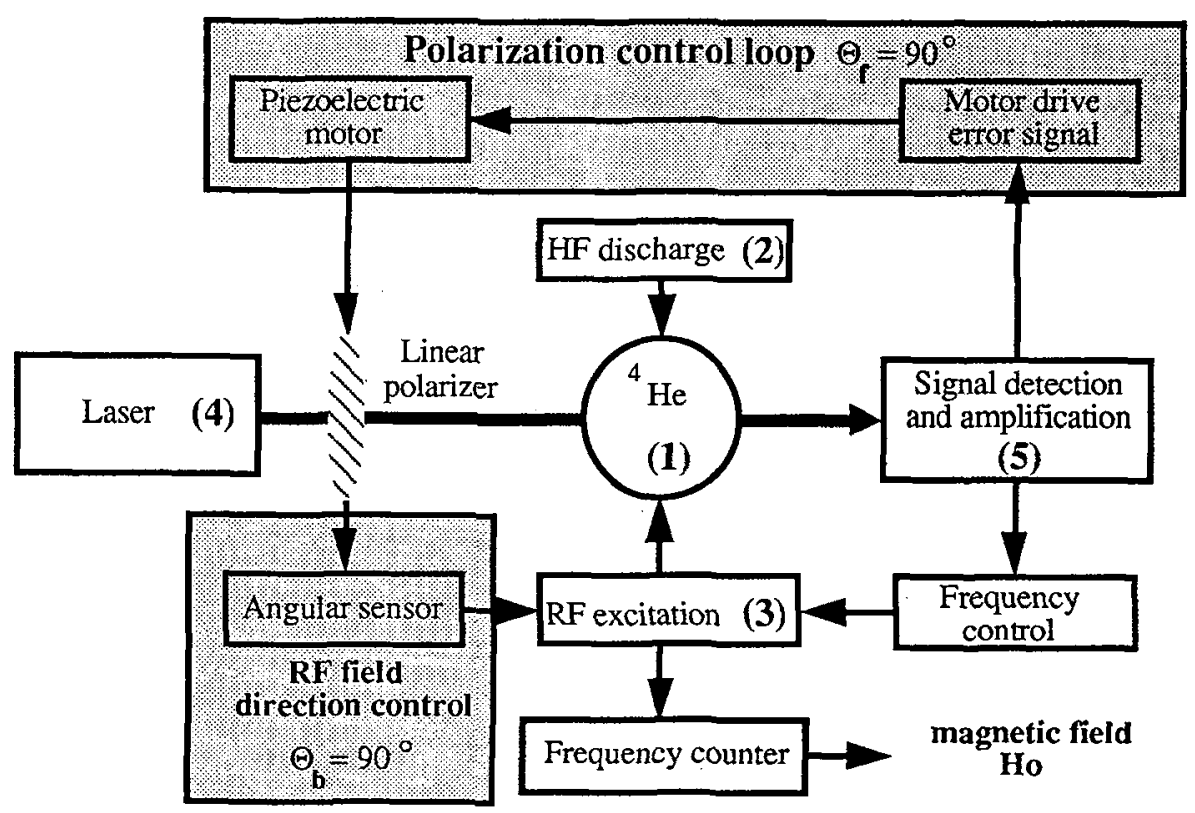

Figure 2 : Isotropic helium magnetometer structure

\section{Isotrony tests of our sensor}

Two sets of tests have been carried out in order to check our sensor isotropy:

- a rotation around the sensor axis $K_{0}$ previously set perpendicular to the static magnetic field $\mathbf{H}_{\mathbf{O}}$ (transverse rotation) to make sure that the polarization and $\mathrm{RF}$ field direction control loops work satisfactorily. The transverse components of any magnetic dipole located in the vicinity of the cell will also be detected thanks to this test.

- a rotation for which the angle between the laser beam direction $K_{0}$ and $\mathbf{H}_{0}$ is varied to estimate the longitudinal component of possible magnetic dipoles attached to the sensor.

As can be seen in figure 3 , the heading errors are inferior to $150 \mathrm{pT}$. Moreover, they are mostly attributable to minor flaws in the RF field direction control loop.
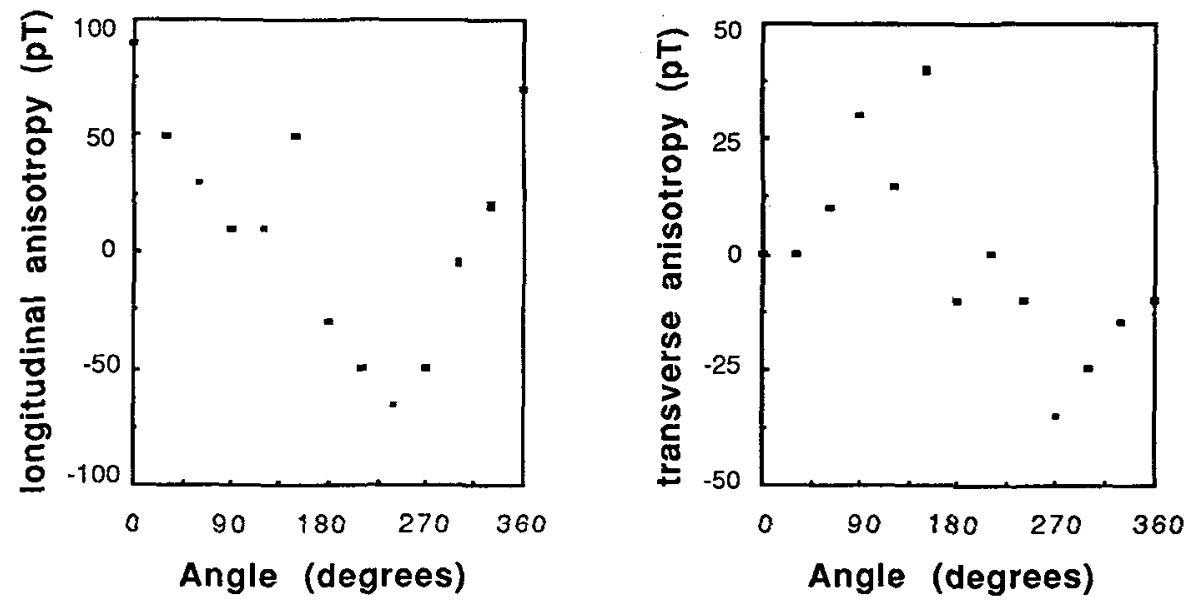


\section{Conclusion}

The new helium magnetometer structure we have designed combines the traditional high sensitivity of optically pumped sensors with a very good isotropy: no amplitude effect has been noticed and the frequency displacements are significantly below the usual values of these devices. Although the isotropy should still be improved after the angular sensor replacement, our magnetometer is already very well adapted for airborne applications.

\section{Acknowledgement}

This work has been supported by the Direction des Recherches Etudes et Techniques.

\section{Bibliography}

[1] A. Kastler, 1950, J.Phys.Rad., Vol.1, 255

[2] R.E. Slocum, L.D. Schearer, P. Tin and R. Marquedant, 1988, J.Appl.Phys., Vol 64, 6615

[3] C. Cohen-Tannoudji,1962, Ann.Phys., Vol 7, 423 \& 469

[4] F. Bloch and A. Siegert , 1940, Phys.Rev., Vol 57, 522

[5] J.P. Heppner, 1963, Space Sci.Rev, Vol 2, 315

[6] R. Duffait, M. Froelicher, R. Jacques and P. Minotti, 1991, Revue Cetehor Informations, $N^{\circ} 94,3$ 